\title{
Langerhans cell histiocytosis followed by folliculotropic mycosis fungoides
}

\author{
Izabela Błażewicz ${ }^{1}$, Małgorzata Sokołowska-Wojdyło', Agnieszka Piekarska², Alicja Sadowska-Klasa², \\ Anna Kowalczyk ${ }^{3}$, Monika Konczalska ${ }^{1}$, Berenika Olszewska ${ }^{1}$, Wioletta Barańska-Rybak ${ }^{1}$, Wojciech Biernat ${ }^{4}$, \\ Roman J. Nowicki ${ }^{1}$
}

\begin{abstract}
'Department of Dermatology, Venereology and Allergology, Medical University of Gdansk, Gdansk, Poland 2Department of Hematology and Transplantology, Medical University Medical University of Gdansk, Gdansk, Poland ${ }^{3}$ Department of Oncology and Radiotherapy, Medical University of Gdansk, Gdansk, Poland ${ }^{4}$ Department of Pathology, Medical University of Gdansk, Gdansk, Poland
\end{abstract}

Adv Dermatol Allergol 2017; XXXIV (3): 273-275 DOI: https://doi.org/10.5114/pdia.2017.67055

The occurrence of lymphoma and Langerhans cell histiocytosis (LCH) in the same individual, either sequentially or simultaneously is not common [1, 2]. Several cases in which malignant lymphoma occurred prior to LCH are reported [3, 4]; however, a few cases can be found with LCH followed by malignant lymphomas [5]. We report a rare accompaniment of LCH followed by folliculotropic mycosis fungoides (FMF). Due to another diagnosis of the patient described previously in Advances of Dermatology and Allergology [6], we would like to present a continuation of her diagnostic and therapeutic process, because it changes the look on the current course of the illness, forcing clinicians to be vigilant. A 41-year-old woman was admitted to the Department of Dermatology, Venereology and Allergology in 2011 due to diffuse edematous skin lesions and numerous nodules localized on the face. The physical examination revealed also a $2 \times 2 \mathrm{~cm}$-sized ulcer covered with necrotic tissue on the skin of the left lower extremity and generalized lymphadenopathy. The diagnostic tests (magnetic resonance imaging (MRI) of the craniofacial area, computed tomography (CT) of the chest, bone marrow biopsy, chest X-ray, immunophenotypic examination of peripheral blood, ultrasonography of lymph nodes) were performed, but they did not reveal any abnormalities. Cervical lymph node biopsy was taken and on the basis of histopathological examination, diagnosis of LCH was made. The neoplastic cells showed the following phenotype: CD1a+, S100-, CD2-, CD3-, CD68-, CD56-, thiaphorin-1-, CD21-, Granzyme $\mathrm{B}-$-, CD5-. The final diagnosis of LCH was based on the clinical picture, histopathological examination of the skin and lymph node. The diagnosis was confirmed by immunohistochemical staining, which revealed the presence of aggregates of CD1a+ cells. Prednisone therapy at the initial dose of $1 \mathrm{mg} / \mathrm{kg} /$ day was started. During the treatment, rapidly growing facial edema occurred, resulting from compression by enlarged carotid lymph nodes on the blood vessels. In the Department of Oncology and Radiotherapy, the chemotherapy with cladribine was initiated ( $5 \mathrm{mg} / \mathrm{m}^{2} /$ day for 5 days every 4-6 weeks). The patient was given 6 courses of chemotherapy with a significant improvement. Unfortunately, 2 months after completion of treatment, papular lesions localized on the trunk occurred. Their histopathological examination revealed $\mathrm{LCH}$. The chemotherapy with vinblastine $6 \mathrm{mg} /$ $\mathrm{m}^{2}$ and prednisone $40 \mathrm{mg} / \mathrm{m}^{2} /$ day was initiated with partial improvement. Due to the progression after 2 years of the disease and as a result of further investigations, the diagnosis was modified. Initially, the coexistence of LCH and peripheral blood T cells lymphoma, and ultimately, after a pathological consultation in the United States of America, folliculotropic mycosis fungoides (FMF) was diagnosed (Figure 1). The patient remained under the care of the Department of Hematology and Transplantology and because of life-threatening disease progression was treated with chemotherapy regimens: CHOED, GDP and after verification of the diagnosis: CHOED+ PUVA-therapy + IFN $\alpha 2$ a (3,000,000 IU 3 times a week) with a very good partial remission. The patient underwent allogeneic hematopoietic cell transplantation (HCT) with total skin and total nodal irradiation, with FMF progression 100 days after HCT. Immunosuppression tapering was complicated with acute graft-versus-host disease (GvHD) grade 3 (skin and gastrointestinal tract involvement), treated successfully with steroids, methotrexate and extracorporeal photopheresis (ECP) performed every week (2 consecutive days), with initial regression of FMF. The ECP was continued twice a month but due to FMF pro-

Address for correspondence: Izabela Błażewicz MD, Department of Dermatology, Venereology and Allergology, Medical University of Gdansk, 1a Kliniczna St, 80-402, Gdansk, Poland, fax: +48791002315, e-mail: izabela.blazewicz@wp.pl Received: 1.05.2016, accepted: 14.06.2016. 

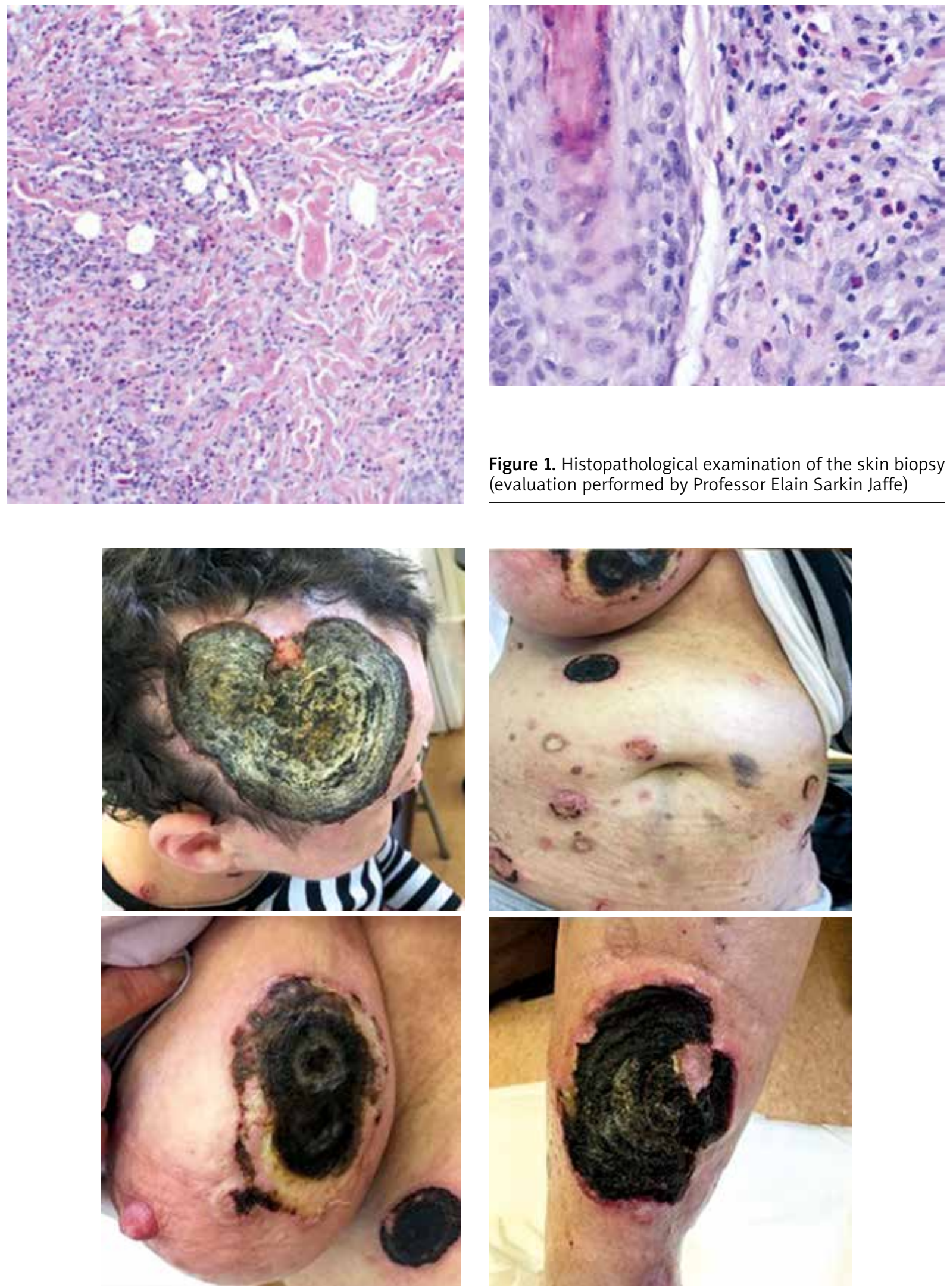

Figure 2. Progression of folliculotropic mycosis fungoides 
gression (Figure 2) bexarotene and IFN $\alpha 2 a(3,000,000 \mathrm{IU}$ 3 times a week), followed by palliative radiotherapy on the scalp and interscapular region, were implemented. Despite the partial remission within the skin, the patient died 36 months after diagnosis due to a septic shock.

Cells of the hematopoietic system are derived from common precursors that differentiate into lineages with distinct morphologic, immunophenotypic, and functional characteristics. The lineage of the cell origin is the major criterion used to classify hematopoietic neoplasms. Langerhans cells (LCS) have been classically considered of myeloid origin, but recent reports, which demonstrate the existence of lymphoid dendritic cells (DCs) derived from multipotent lymphoid precursors devoid of myeloid differentiation potential, raise the question of the lymphoid or myeloid origin of LCs [7]. Some clinical data have shown that two hematopoietic populations in the same patient may share identical genetic changes or abnormalities, raising the possibility that tumors expressing the phenotype of one hematopoietic lineage might "transdifferentiate" into a genetically similar but phenotypically distinct tumor of a different lineage [3, 4]. Langerhans cell histiocytosis has a complex association with malignant lymphoma: it may precede, occur with, or follow it [8]. A clonal relationship between histiocytic/ dendritic cell neoplasms and lymphoid malignancies was first suggested by Wetzler et al. [9]. Common clonal origins were subsequently demonstrated by molecular genetic techniques between precursor T-lymphoblastic lymphoma/leukemia and histiocytic sarcoma [10], Langerhans cell histiocytosis [1], and most recently, follicular lymphoma and histiocytic/dendritic neoplasms [3, 4]. We reported a patient in whom $\mathrm{LCH}$ was diagnosed and 3 years later FMF was seen as well. Some physicians may assume that these two disorders may have occurred concurrently, and one was not diagnosed. The arguments confirming the primary occurrence of $\mathrm{LCH}$ are: initial good response to LCH therapy alone for 2 years, the result of histopathological examination of the skin and lymph node, and also the result of immunohistochemical staining, which revealed the presence of aggregates of CD1a+ cells. Feldman et al. reported a series of 8 patients with both follicular lymphomas and histiocytic/dendritic cells (H/DC) tumors, and provided evidence for the first time of the same underlying molecular oncogenetic alteration in both neoplasms, but in no case the H/DC tumor preceded the diagnosis of FL [4]. According to our knowledge, it is the first report in which the diagnosis of $\mathrm{LCH}$ preceded FMF. It is important to consider the probability of occurrence of these two conditions in the same patient and using an appropriate technique to differentiate them to avoid misdiagnosis. An accurate diagnosis in such situations helps the physician to choose the appropriate therapy.

\section{Acknowledgments}

The authors would like to thank Professor Elain Sarkin Jaffe, President of the Society of Hematopathology, for the histopathological analysis of skin and lymph nodes biopsies, which led to the final diagnosis and implementation of the targeted therapy.

\section{Conflict of interest}

The authors declare no conflict of interest.

\section{References}

1. Feldman AL, Berthold F, Arceci RJ, et al. Clonal relationship between precursor T-lymphoblastic leukaemia/lymphoma and Langerhans-cell histiocytosis. Lancet Oncol 2005; 6: 435-7.

2. Fraser CR, Wang W, Gomez M, et al. Transformation of chronic lymphocytic leukemia/small lymphocytic lymphoma to interdigitating dendritic cell sarcoma: evidence for transdifferentiation of the lymphoma clone. Am J Clin Pathol 2009; 132: 928-39.

3. Zhang D, McGuirk J, Ganguly S, et al. Histiocytic/dendritic cell sarcoma arising from follicular lymphoma involving the bone: a case report and review of literature. Int I Hematol 2009; 89: 529-32.

4. Feldman AL, Arber DA, Pittaluga S, et al. Clonally related follicular lymphomas and histiocytic/dendritic cell sarcomas: evidence for transdifferentiation of the follicular lymphoma clone. Blood 2008; 111: 5433-9.

5. Safaei A, Bagheri M, Shahryari J. Langerhans cell histiocytosis followed by Hodgkin lymphoma: a case report. Iran J Med Sci 2015; 40: 282-6.

6. Błażewicz I, Biernat W, Kowalczyk A, et al. Adult onset of multisystem Langerhans cell histiocytosis with skin and lymph node involvement. Postep Dermatol Alergol 2015; 32: 225-8.

7. Régis A, Ben Salem D, Lambert A, et al. Concomitant pulmonary Langerhans cell histiocytosis and malignant lymphoma: report of two cases. J Radiol 2009; 90: 66-8.

8. Egeler RM, Neglia JP, Puccetti DM, et al. Association of Langerhans cell histiocytosis with malignant neoplasms. Cancer 1993; 71: 865-73.

9. Wetzler M, Kurzrock R, Goodacre AM, et al. Transformation of chronic lymphocytic leukemia to lymphoma of true histiocytic type. Cancer 1995; 76: 609-17.

10. Feldman AL, Minniti C, Santi M, et al. Histiocytic sarcoma after acute lymphoblastic leukaemia: a common clonal origin. Lancet Oncol 2004; 5: 248-50. 